\title{
Determinación directa de mercurio en sangre y orina HR CS GFAAS con AuNPs como modificante químico
}

\author{
Diego Pereira Leite $^{\mathrm{A}}$, Ananda Guarda ${ }^{\mathrm{A}}$, Maite Aramendía ${ }^{\mathrm{A}, \mathrm{B}}$, Martín Resano $^{\mathrm{A}}$ \\ A Groupo de Métodos de Análisis Rápidos con Técnicas Espectroscópicas (M.A.R.T.E.) - Departamento \\ de Química Analítica, Instituto Universitario de Investigación de Ingeniería en Aragón (I3A), \\ Universidad de Zaragoza, Pedro Cerbuna 12, 50009 Zaragoza, España \\ ${ }^{\mathrm{B}}$ Centro Universitario de la Defensa de Zaragoza, Academia General Militar de Zaragoza, Carretera de \\ Huesca s/n, 50090 Zaragoza, España
}

\begin{abstract}
Este trabajo usa la Espectrometría de Absorción Atómica con Horno de Grafito (HR CS GFAAS) para cuantificación de mercurio (Hg) en muestras certificadas (CRM) de orina y sangre, usando nanopartículas de oro (AuNPs) como modificante químico. Los porcentajes de recuperación obtenidos de muestras de CRM concuerdan con los valores de referencia (86 al 100\%), presentando además valores de incertidumbre similares.
\end{abstract}

\section{Introducción}

El mercurio es un contaminante que despierta una preocupación global por su conocida toxicidad al ser humano y al ecosistema, y por su capacidad de moverse fácilmente por la atmósfera, debido a su volatilidad $^{1}$. La exposición humana al $\mathrm{Hg}$ elemental está reflejada principalmente en la orina, siendo el biomarcador más empleado para monitorizar exposiciones, aunque la sangre también sea comúnmente utilizada ${ }^{1}$. En España, el Instituto Nacional para la Salud y Seguridad Ocupacional (INSHT) ha delimitado los valores de exposición del Hg a $40 \mu \mathrm{L}^{-1}$ en orina y 10 $\mu \mathrm{L}^{-1}$ en sangre ${ }^{2}$.

En ese contexto, el uso de HR CS GFAAS se presenta como una alternativa viable. Esta técnica ofrece sensibilidad adecuada para la determinación elemental a niveles traza, permitiendo análisis de sólidos y matrices complejas (tales como sangre y orina), además de ofrecer bajo coste de mantenimiento y operación ${ }^{3}$. No obstante, la determinación de $\mathrm{Hg}$ por GFAAS no es trivial, debido a su baja sensibilidad y estabilidad térmica. Estos problemas pueden ser parcialmente compensados al ejecutarse el análisis directo de sólidos en el horno de grafito, donde se evitan las diluciones y se mejora la sensibilidad. Por otra parte, el uso de modificantes químicos, tales como $\mathrm{KMnO}_{4}$ y $\mathrm{Pd}$, permite la estabilización térmica del analito. En ese trabajo, son se han explorado estrategias para la determinación directa de $\mathrm{Hg}$ en muestras de sangre $y$ orina a concentraciones traza, evitando cualquier tipo preparación de muestras, minimizándose los riesgos de contaminación y pérdidas del analito. Para la estabilización térmica del $\mathrm{Hg}$, se propone el uso de nanopartículas de oro (AuNPs) como modificante químico, fácilmente sintetizables en laboratorio ${ }^{4}$.

\section{Experimental}

Se sintetizaron en laboratorio AuNPs de 20 nm por el método de Turkevich. Se utilizaron CRMs de sangre y orina para la determinación de Hg, cuyas concentraciones eran de 17.3 y $39,5 \mu \mathrm{g} \mathrm{L}^{-1}$ en orina y 16,0 y $37,1 \mu \mathrm{g} \mathrm{L}^{-1}$ en sangre. Para los análisis de Hg en sangre y orina se usó un espectrómetro de absorción atómica de fuente continua de alta resolución, modelo ContrAA 700 (Analytik Jena,), equipado con atomizador de horno de grafito.

\section{Resultados}

Primeramente, se optimizó el programa de calentamiento del GF AAS con diversos modificantes químicos, de los cuales las 
AuNPs son las que mejor estabilizaron térmicamente el Hg (Figura 1).

La validación del método desarrollado empleando AuNPs como modificante químico se realizó mediante análisis de CRM de sangre y orina. En consecuencia, se verificó la exactitud y la precisión del método con calibración con patrones acuosos de Hg. Los resultados obtenidos pueden se encuentran en la Tabla 1 , donde está presentada la mediana de las medidas para cada muestra, con su respectivo intervalo de confianza al $95 \%$ para 5 medidas.

\section{Conclusiones}

El método presentó buenos valores de recuperación (86 a 100\%) para las muestras de CRM, con intervalos de confianza comparables a los valores de referencia. De este modo, el método desarrollado puede ser aplicado para la determinación de Hg en muestras de sangre y orina por HR CS GFAAS.

\section{Referencias}

[1] Evers D.C.; Keane S.E.; Basu N.; Buck D. Sci Total Environ. 2016, 569-570, 888-903.

[2] INSHT, 2016

[3] Aramendía, M., et al. JAAS, 2011, 1964.

[4] Resano, M.; Briceño, J.; Belarra, M.A. Spectrochimica Acta Part B, 2009, 520 - 529.

Figura 1: Curvas de pirolisis y atomización de Hg (5 ng) sin adición de modificante químico y en presencia de los siguientes modificantes: AuNPs (500 ng de una suspensión acuosa de NPs con diámetro de $10 \mathrm{~nm}), \mathrm{KMnO}_{4}(300 \mu \mathrm{g}), \mathrm{Pd}\left(10 \mu \mathrm{g}\right.$ adicionados como $\left.\mathrm{Pd}\left(\mathrm{NO}_{3}\right)_{2}\right)$ y $\mathrm{Au}(\mathrm{III})(10 \mu \mathrm{g}$ adicionados como $\mathrm{HAuCl}_{4}$ ).

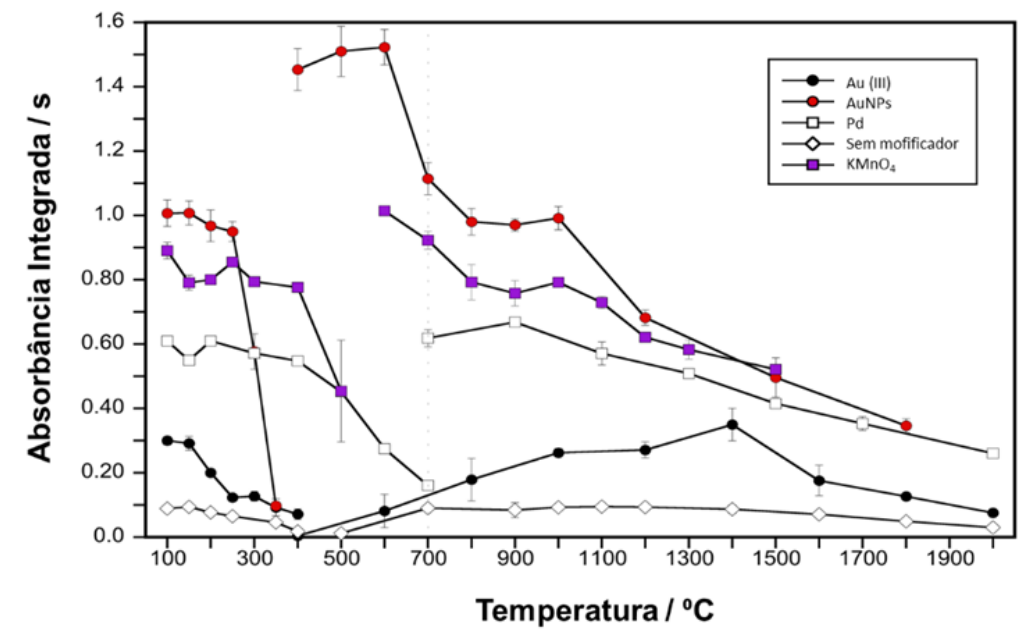

Tabla 1. Determinación de Hg por HR CS GFAAS en muestras certificadas de orina y sangre $(n=5)$.

\begin{tabular}{|c|c|c|c|}
\hline Muestra & Valor Certificado $\left(\mu g \mathbf{L}^{-1}\right)$ & Valor obtenido $\left(\mu \mathrm{gL}^{-1}\right)$ & Recuperación \\
\hline Urina - Clincheck Level II Lote 923 & $39,5 \pm 7,5$ & $35,8 \pm 2,8( \pm 7,8 \%)$ & $90 \%$ \\
\hline Urina - Clincheck Level II Lote 432 & $17,3 \pm 4,3$ & $15,0 \pm 1,9( \pm 12,6 \%)$ & $86 \%$ \\
\hline Sangue - - Level III & $37,1 \pm 7,5$ & $36,0 \pm 4,4( \pm 12,2 \%)$ & $97 \%$ \\
\hline Sangue - Level II & $16,0 \pm 3,2$ & $16,0 \pm 2,4( \pm 15,0 \%)$ & $100 \%$ \\
\hline
\end{tabular}

